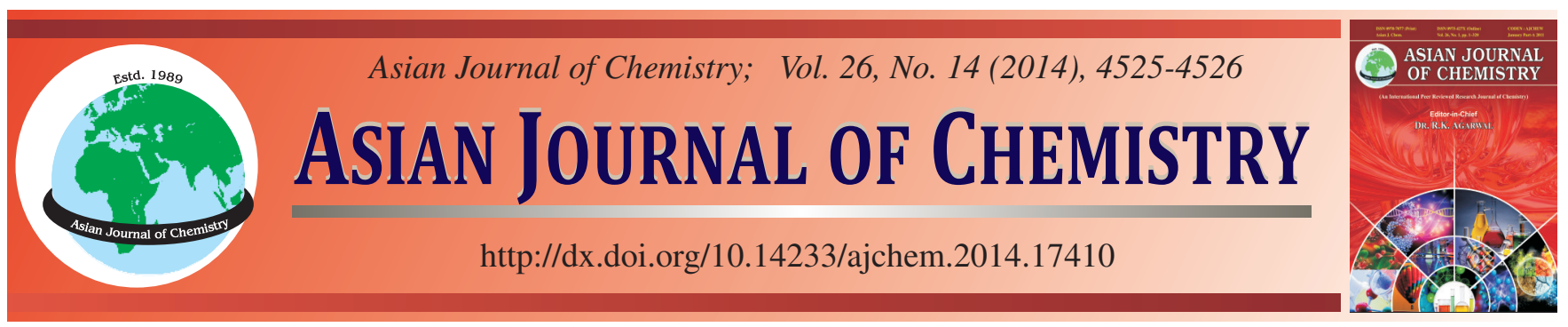

NOTE

\title{
Hydrothermal Synthesis and Crystal Structure of Co(II) Coordination Polymer
}

\author{
ZePeng Chu and Minghui Zhao*
}

Shandong Academy of Building Research, Shandong 250031, P.R. China

*Corresponding author: E-mail: shdyzhang@126.com

Received: 18 February 2014;

Accepted: 20 April 2014;

Published online: 5 July 2014;

AJC-15514

\begin{abstract}
A new coordination polymer $[\mathrm{Co}(\mathrm{BDC})(\text { bimb })]_{\mathrm{n}}(1)$ with 1,3-benzene dicarboxylic acid $\left(\mathrm{H}_{2} \mathrm{BDC}\right)$ and 1,4-bis(1-imidazol-yl)-2,5-dimethyl benzene (bimb) has been has been prepared by hydrothermal synthesis and characterized by single-crystal X-ray diffraction and EA. Complex, $[\mathrm{Co}(\mathrm{BDC})(\mathrm{bimb})]_{\mathrm{n}}(1)$, is triclinic, space group P-1 with $\mathrm{a}=8.792(5), \mathrm{b}=10.076(5), \mathrm{c}=11.767(5) \AA, \alpha=85.577(5), \beta=$ $82.481(5)^{\circ}, \gamma=74.942(5), \mathrm{V}=997.0(9) \AA^{3}, \mathrm{Z}=2, \mathrm{Mr}=461.33, \mathrm{Dc}=1.537 \mathrm{~g} / \mathrm{cm}^{3}$ and $\mathrm{F}(000)=474$. The final refinement gave $\mathrm{R}=$ 0.0411 and $w R=0.0978$ for 3634 reflections with $\mathrm{I}>2 \sigma(\mathrm{I})$. X-ray diffraction analysis reveals that the complex 1 displays a twodimensional structure with $(4,4)$-connected $s q l$ topology.
\end{abstract}

Keywords: Coordination polymer, Co (II), Crystal structure.

ᄂ - - - - - - - - - - - - - - - - - - - - - - - -

The field of metal-organic frameworks (MOFs) has grown rapidly in recent years, in part due to their ease of preparation, tunability and wide range of potential applications in areas such as gas storage, separation and catalysis and most recently drug delivery ${ }^{1-4}$. We have a long-standing program of research in this area for the preparation of metal-organic frameworks using multiple ligands of dissimilar charge $\mathrm{e}^{5-8}$. The use of mixed ligands to prepare metal-organic framework materials is well studied since combining organic ligands of different connectivity, charge and donor functionality introduces additional levels of structural complexity into the resulting frameworks, promising access to materials with enhanced properties and applications. A powerful strategy to identify and prepare new complex structural topologies is the combination of small structure-directing agents (SDAs) or template molecules with mixed ligand systems ${ }^{9,10}$.

All the reagents and solvents employed were commercially available and used without further purification. Elemental analysis was carried out on a Carlo Erba 1106 full-automatic trace organic elemental analyzer.

Synthesis of $[\mathbf{C o}(\mathbf{B D C})(\mathbf{b i m b})]_{\mathbf{n}}(\mathbf{1})$ : The mixtures of $\mathrm{CoCl}_{2} \cdot 6 \mathrm{H}_{2} \mathrm{O}$ (0.5 mmol, $\left.0.119 \mathrm{~g}\right)$, 1,3-benzene dicarboxylic acid ( $\left.\mathrm{H}_{2} \mathrm{BDC}\right)$ (0.5 mmol, $\left.0.083 \mathrm{~g}\right), 1,4-b i s(1-i m i d a z o l-y l)-$ 2,5-dimethyl benzene (bimb) (0.5 mmol, $0.119 \mathrm{~g}), \mathrm{NaOH}$ (1 mmol, $0.04 \mathrm{~g}$ ) and $12 \mathrm{~mL}$ of water were heated to $140{ }^{\circ} \mathrm{C}$ for 4 days and then cooled to room-temperature. The red crystals were obtained in pure phase, washed with water and ethanol and dried at room temperature (Yield: $32 \%$ based on Co).
Elemental Anal. Calcd. (\%) for $\mathrm{C}_{22} \mathrm{H}_{18} \mathrm{~N}_{4} \mathrm{O}_{4} \mathrm{Co}$ : C, 57.28; $\mathrm{H}$, 3.93; N, 12.14. Found: C, 57.29; H, 3.94; N, 12.15 .

X-ray crystallography: Single crystal X-ray diffraction analyses of complex $\mathbf{1}$ was carried out on a Bruker SMART APEXII CCD diffractometer equipped with a graphite monochromated $\mathrm{MoK}_{\alpha}$ radiation ( $\lambda=0.71073 \AA$ ) by using a $\omega$-scan mode. Empirical absorption correction was applied using the SADABS programs ${ }^{11}$. All the structures were solved by direct methods and refined by full-matrix least-squares methods on $\mathrm{F}^{2}$ using the program SHEXL $97^{12}$. All non-hydrogen atoms were refined anisotropically. The hydrogen atoms were located by geometrically calculations and their positions and thermal parameters were fixed during the structure refinement (Fig. 1).

The X-ray crystallographic study reveals that complex $\mathbf{1}$ crystallized in the triclinic system, P-1 space group. Each independent $\mathrm{Co}(\mathrm{II})$ is located at the inversion center and coordinated by two nitrogen atoms $[\mathrm{Co}(1)-\mathrm{N}(1)=2.131(2) \AA$ and Co (1)-N (4) = 2.135(2) $\AA$ ] and four carboxylate oxygen atoms [Co-O, ranging from 2.031(2) $\AA$ to 2.342(2) $\AA$ ] to furnish an octahedral geometry (Fig. 2).

The axis positions are occupied by two nitrogen atoms and the equatorial plane is defined by four carboxylate atoms. The carboxylate ligands connect the $\mathrm{Co}$ (II) to form a onedimensional chain. In 1D chain, the $\mu^{2}-\eta^{1}: \eta^{1}$ carboxylate group link two $\mathrm{Co}(\mathrm{II})$ atoms to construct a dinuclear $\mathrm{Co}$ (II) unit with the Co...Co distance of $4.329 \AA$. The 1D chains are further connected by bimb ligands to construct a two-dimensional layer structure (Fig. 3). 


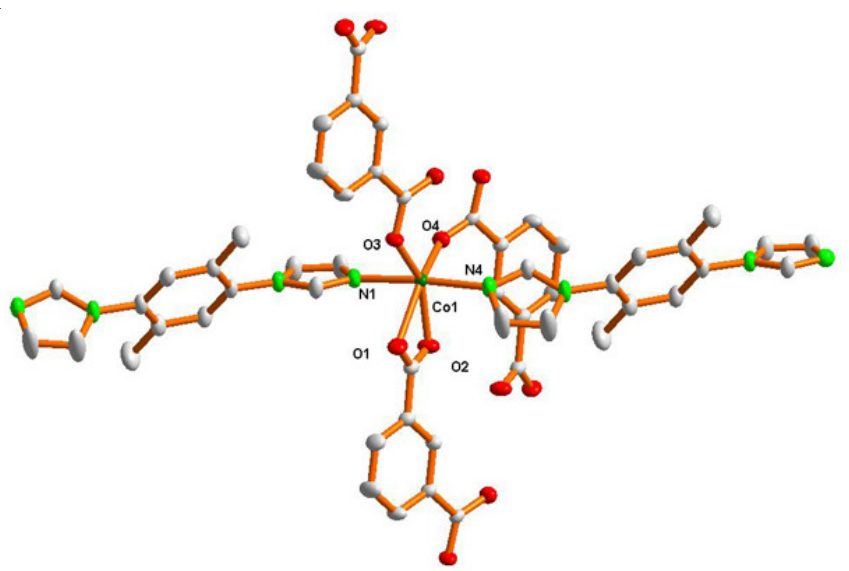

Fig. 1. Coordination environment for $\mathrm{Co}(\mathrm{II})$ ion

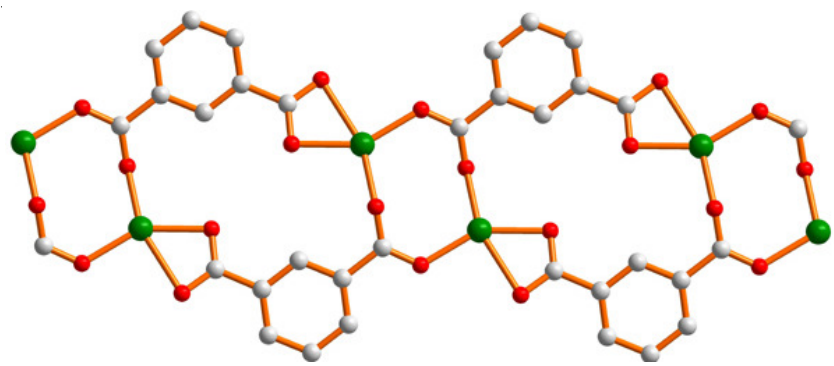

Fig. 2. 1D chain for complex 1 constructed by Ni(II) ions and carboxylate ligands

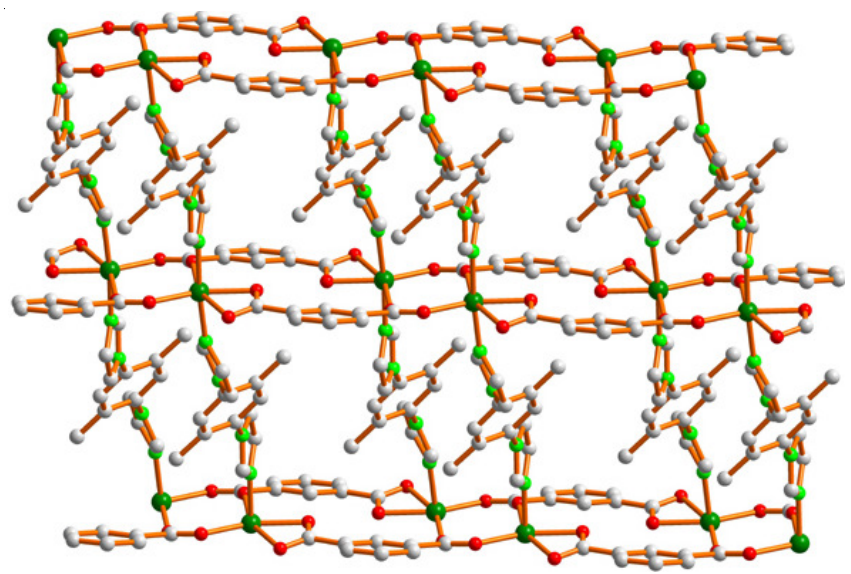

Fig. 3. 2D layer structure for complex 1

\section{REFERENCES}

1. C. Janiak, Dalton Trans., 2781 (2003).

2. X. Lin, J.H. Jia, X.B. Zhao, K.M. Thomas, A.J. Blake, G.S. Walker, N.R. Champness, P. Hubberstey and M. Schroder, Angew. Chem. Int. Ed., 45, 7358 (2006).

3. M. Eddaoudi, J. Kim, N. Rosi, D. Vodak, J. Wachter, M. O'Keefe and O.M. Yaghi, Science, 295, 469 (2002).

4. B. Rather and M.J. Zaworotko, Chem. Commun., 830 (2003).

5. F. Guo, Inorg. Chim. Acta, 399, 79 (2013).

6. G.L. Xu and F. Guo, Inorg. Chem. Commun., 27, 146 (2013).

7. J. Li, J. Tao, R.B. Huang and L.S. Zheng, Inorg. Chem., 51, 5988 (2012).

8. A.G. Wong-Foy, A.J. Matzger and O.M. Yaghi, J. Am. Chem. Soc., 128, 3494 (2006).

9. C. Serre, F. Millange, C. Thouvenot, N. Gardant, F. Pellé and G. Férey, J. Mater. Chem., 14, 1540 (2004).

10. F. Guo, X.L. Zhang, B.Y. Zhu and J.C. Qiu, J. Inorg. Organomet. Polym., 20, 38 (2010).

11. SAINT Software Reference Manual; Bruker AXS: Madison, WI (1998).

12. G.M. Sheldrick, SHELXTL NT, version 5.1; Program for Solution and Refinement of Crystal Structures, University of Göttingen, Germany (1997). 